\title{
WLAN Uygulamaları için Düşük Gürülttülü Kuvvetlendirici Tasarımı
}

\author{
Aysu Belen ${ }^{1 *}$ \\ 1* İskenderun Teknik Üniversitesi, İskenderun meslek Yüksek Okulu, Hibrid ve Elektrikli Araçlar Bölümü, Hatay, Türkiye (ORCID: 0000-0001-5038-424X), \\ aysu.belen@iste.edu.tr
}

(İlk Geliş Tarihi 19 Nisan 2021 ve Kabul Tarihi 8 Ağustos 2021)

(DOI: $10.31590 /$ ejosat.920045)

ATIF/REFERENCE: Belen, A. (2021). WLAN Uygulamaları için Düşük Gürültülü Kuvvetlendirici Tasarımı. Avrupa Bilim ve Teknoloji Dergisi, (25), 665-668.

\begin{abstract}
Öz
Kablosuz haberleşme sistemlerinin gelişmesiyle birlikte bu alanda kullanılacak sistemlerin alt birleşenlerine olan talep giderek artmaktadır. Alıcı sistemlerde düşük gürültülü kuvvetlendirici (LNA) anahtar rol oynamaktadırlar. Bu elemanlar sistemin genel performansında önemli bir rol oynamaktadırlar. Sistem girişindeki düşük seviyedeki sinyalleri alarak yükseltirler bu aşamada olabildiğince düşük gürültü seviyesine sahip olması önemlidir. Bu çalışma kapsamında 5.6GHz WLAN uygulamaları ile uyumlu düşük gürültülü kuvvetlendirici (LNA) tasarımı ve üretimi gerçekleştirilmiştir. BFP720 SiGe transistörlü LNA tasarımı yapılarak sistem içindeki performansı incelenmiş ve sağladığı yüksek kazanç yanı sıra yüksek kesim voltajına olanak sağladığı için bu transistörün kullanımına karar verilmiştir. Tasarımda FR4 taban malzemesi olarak seçilmiştir. Tasarımlarda AWR microwave Office yüksek frekans programı kullanılmıştır. Yapılan ölçümlerde transistör 2.8V ve $10 \mathrm{~mA}$ ile beslenerek $5.6 \mathrm{GHz}$ bandında $13 \mathrm{~dB}$ kazanç, $\mathrm{S}_{11}<-10 \mathrm{~dB}$ elde edilmiştir.
\end{abstract}

Anahtar Kelimeler: LNA, Düşük Gürültülü Kuvvetlendirici, WLAN, BFP720.

\section{Low Noise Amplifier Design for WLAN Applications}

\begin{abstract}
With the recent rapid development in wireless communication systems the demands to its sub-systems also had increased. One of the key element in sub-systems is Low Noise Amplifier (LNA) stage. The performance of this stage has the highest effect on the overall performance of the whole system. Amplification of the low amplitude input signals with the possible lowest noise is at most importance. Herein, design and its realization of a high performance, low cost, and wideband Low Noise Amlifier (LNA) for 5.6 GHz WLAN application had been taken into the study. BFP720 SiGe had studied and determined as an optimal transistor for the aimed LNA design due to its high gain and cut-off voltage values. The aimed LNA design had been simulated in AWR microwave Office high frequency simulator using FR4 material. Based on the experimental results, with a DC biased condition of $2.8 \mathrm{~V} 10 \mathrm{~mA}$, the designed achieves a gain of $13 \mathrm{~dB}$ with $\mathrm{S}_{11}$ value of less than $-10 \mathrm{~dB}$ at $5.6 \mathrm{GHz}$
\end{abstract}

Keywords: LNA, Low Noise Amplifier, WLAN, BFP720.

\footnotetext{
*Sorumlu Yazar: aysu.belen@iste.edu.tr
} 


\section{Giriş}

Günümüzde teknolojinin hızlı büyümesiyle birlikte, yeni nesil haberleşme sistemlerinde daha geniş bant talebi ortaya çıkmıştır. Yeni nesil haberleşme sistemlerinde yaşanan bu talep bilgiye kesintisiz ve hızlı erişim isteğinde kaynaklanmaktadır. Kablosuz haberleşme sistemlerindeki taşınabilir cihaz sayısının ve çeşitliliğinin artması söz konusu cihazların performansında ve hassasiyetini önemli hale getirmiştir. Bütünleşmiş devre yapıları ile gerçekleştiriliyor olmaları, düşük maliyetleri, hızlı veri alışverişine uygun kablosuz haberleşme protokolü olan IEEE 802.11b/g, 2.4-2.5 GHz bandında geniş bir kullanım alanına sahiptirler. Bu nedenle kablosuz yerel alan ağ (WLAN) (Ulrich 2000) sistemlerinin, sivil ve askeri uygulamalar gibi çok geniş banda sahip sistemlerin tasarım ve incelemesi ciddi bir araştırma konusudur (David 1998), (Klude ve Dathe 2003), (Akyildiz 2002), (Alaybeyoğlu 2009). Alıcı verici sistemlere olan bu eğilimin sürekli artması nedeniyle; özellikle tüketiciler, düşük maliyetli, düşük güç tüketimin sahip (mobil ve taşınabilir ürünler), küçük boyutlar istemektedirler (Esame ve Kaynak 2006).

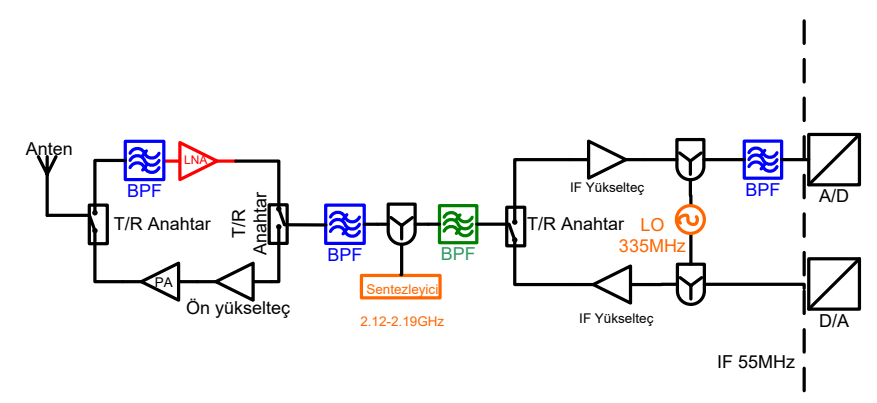

Sekil 1: RF Alıcl-Verici Blok Diyagramı

Şekil 1'de haberleşme sistemleri için alıcı verici blok diagramı verilmiştir. Ortamdan anten vasıtasıyla alınan elektromanyetik dalgalar RF ön uç modüle aktarılarak analog sinyal temel band sinyal formuna dönüştürülerek dijital dönüştürme kısmına getirilir. Alıcı kısmında blok şemasında görüldüğü üzere anten yer almaktadır son yıllarda antenler üzerine literatürde bir çok yenilikçi çalışmaya bulunmaktadır Duyarlılık ve seçicilik parametreleri RF ön uç modüllerde alıcı tasarımında göz önünde bulundurulması gereken önemli parametreler arasındadır (Çalışkan 2019), (Koçer ve Aydemir 2020), (Danacı ve Palandöken). LNA katı alıcı kısımdaki en önemli tasarım katı olmakla birlikte alıcı kısmın hassasiyetini belirlemektedir. Bu çalışma kapsamında RF ön uç modüller için, WLAN uygulamalarında kullanılmak üzere LNA tasarımı, üretimi ve ölçümleri gerçekleştirilmiştir.

\section{RF Yükselteç Tasarımı}

Gelişen kablosuz haberleşme sistemleri uygulamaları, farklı haberleşme protokolleri arasındaki geçişlerin kolay olmasını gerektirmektedir. Bu durum çoklu standartlara sahip alıcıların tasarlanmasına neden olmuştur. Şekil 1'de sayısal haberleşme sistemleri için alıcı verici blok diagram gösterilmiştir. Alıcı kısımda en önemli blok düşük gürültülü yükselteç bloğudur. Alıcı kısımda, ortamdan alınan sinyal seviyesinin çok düşük olmasından dolayı bu sinyallerin herhangi bir işlem yapılmadan önce düzgün olarak yükseltilmeleri gerekmektedir. Bunun nedeni yükseltme işleminde gürültü seviyesinin ana sinyali geçmesidir. $\mathrm{Bu}$ durumda alınan sinyal bastırılmış olacaktır. $\mathrm{Bu}$ nedenle düşük gürültülü kuvvetlendirici bloğunun gürültü katkısının minimize ederken, alınan sinyali olabildiğince yükseltmesi istenmektedir. Çünkü alıcının ilk kademesinin gürültü faktörü, devrenin toplam gürültü faktörünü baskın şekilde etkiler. Bir diğer önemli hususta düşük gürültü hedeflenirken, giriş empedanlarınında 50 ohm ile uyumlandırılmasını sağlamaktır (Hashemi ve Hajimiri 2002), (Hove ve Faaborg 2004). Küçük sinyal kuvvetlendiricisi tasarlarken seçilen transistör modelinin S-parametresi dosyası üretici firmalardan temin edilebilmektedir (Doddamanş ve Harischandra 2007), (Esame ve Kaynak 2006), (Stece,1999). Bu parametrelerin yanında kararlılık parametreside sistem için önemli bir parametredir (Demirel 2017), (Mahouti 2012).

Bu çalışmada saçılım parametreleri, gürültü şekli, kararlılık, $1 \mathrm{~dB}$ sıkıştırma noktası gibi önemli LNA tasarım parametreleri göz önünde bulundurularak başarılı başarılı bir yükselteç yapılması amaçlanmıştır.

\section{WLAN Uygulamaları için LNA Tasarımı}

Endüstirye uygulamalar için RF derve tasarımı için çeşitli yazılım paketleri bulunmaktadır. Tasarım esnekliği ve çözüm metodu nedeniyle Advancing the Wireless Revoluation Microwave Office (AWR Office) programı kullanılmıştır. $5.6 \mathrm{GHz}$ frekans bandı WLAN uygulamaları için düşük gürültülü kuvvetlendirici tasarımı yapılmıştır. Tasarımda infenion firması tarafindan üretilen BFP720 SiGe transistörü (infinion) kullanılmıştır. $\mathrm{Bu}$ transistörün seçilmesinin sebebi yüksek geçiş frekansına (ft):45GHz, düşük gürültü şekline sahip olması ve yüksek kazanca imkân vermesidir. LNA tasarımında dielektrik sabiti 4.6 , yüksekliği $1.58 \mathrm{~mm}$ olan FR4 alt taban malzemesi kullanılmıştır.

Şekil 2'de tasarlanan devrenin şematik gösterimi sunulmuştur. Devre tasarımında kullanılan R-L-C elemanlarının değerleri tablo 1 'de verilmiştir.

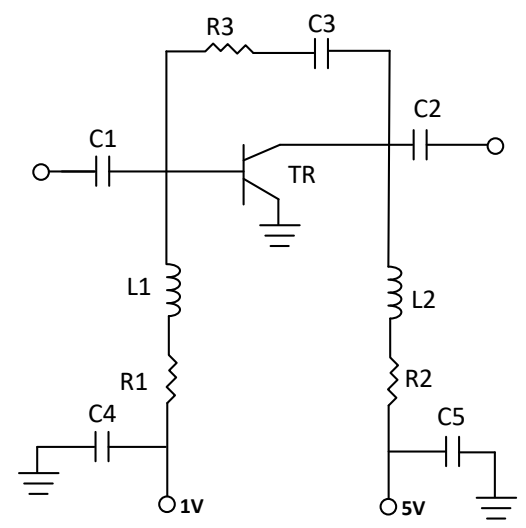

Şekil.2 WLAN Uygulamaları için Tasarlanan LNA şematik gösterimi

Tablo 1. Mazleme parametrleri

\begin{tabular}{|c|c|c|c|}
\hline $\mathrm{C} 1$ & $330 \mathrm{pF}$ & $\mathrm{R} 1$ & $1.5 \mathrm{Kohm}$ \\
\hline $\mathrm{C} 2$ & $330 \mathrm{pF}$ & $\mathrm{R} 2$ & $100 \mathrm{ohm}$ \\
\hline $\mathrm{C} 3-\mathrm{C} 4-\mathrm{C} 5$ & $330 \mathrm{nF}$ & $\mathrm{R} 3$ & $3.9 \mathrm{Kohm}$ \\
\hline $\mathrm{L} 1$ & $33 \mathrm{nH}$ & $\mathrm{L} 2$ & $59 \mathrm{nH}$ \\
\hline
\end{tabular}

Şekil 3(a)'da tasarlanan LNA devresinin saçılma parametreleri verilmiştir. Devrenin $S_{11}$ ve $S_{22}$ değerleri $-15 d B$ altında elde edilmiştir. $S_{21}$ iletim parametresi $14.3 \mathrm{~dB}$ ve $S_{12}$ izolasyon parametresi ise $-15 \mathrm{~dB}$ olarak simülasyonlarda elde edilmiştir. Tasarlanan devrenin gürültü şekli (NF) şekil 3 (b)'de $5.6 \mathrm{GHz}$ de $1.87 \mathrm{~dB}$ olarak elde edilmiştir. 


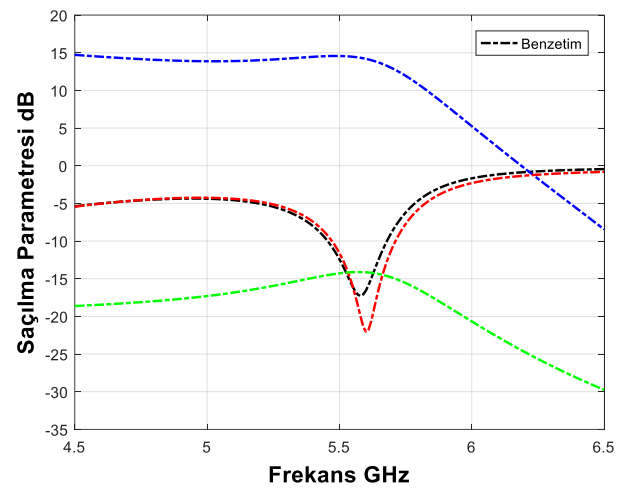

(a)

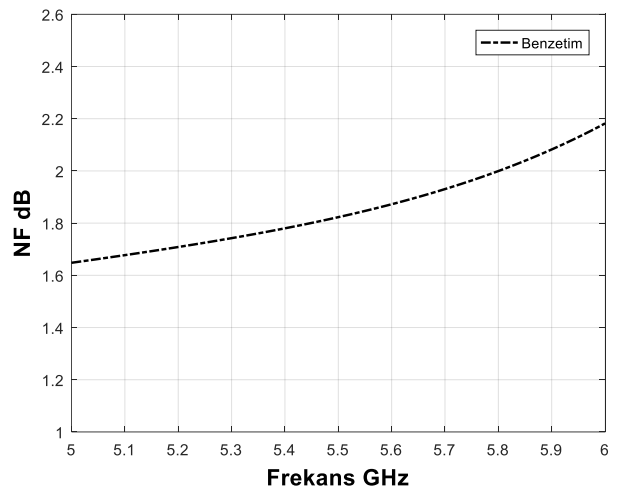

(b)

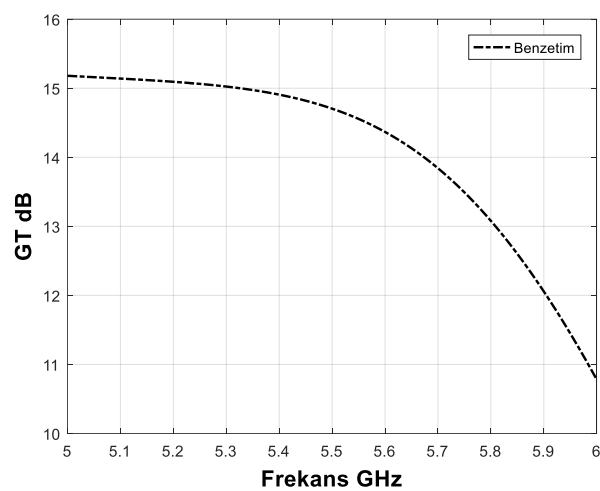

(c)

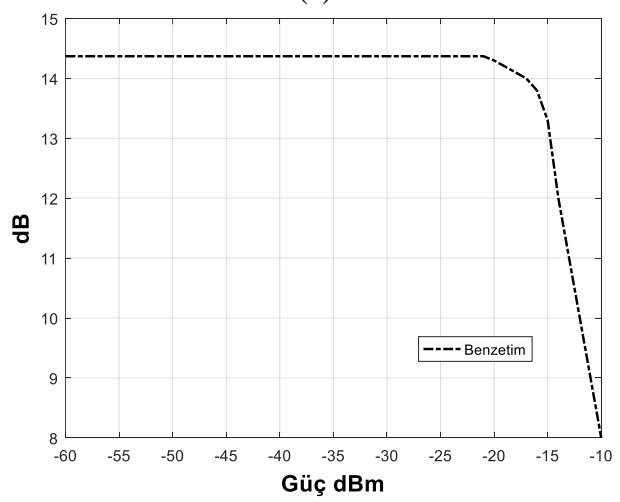

(d)

Şekil.3 Tasarlanan LNA (a) S Parametreleri (b) Gürültü Şekli (c) Kazanç (d) 1 dB Sikıştırma Noktası

Şekil 2'de şematik gösterimi verilen LNA devresinin kazanç benzetim sonucu şekil 3(c)'de $14.3 \mathrm{~dB}$ olarak elde edilmiştir ve $1 \mathrm{~dB}$ sıkıştırma noktası simülasyon sonuçları şekil 3(d)'de $18 \mathrm{dBm}$ olarak elde edilmiştir.

\section{WLAN Uygulamaları için LNA Üretimi}

$5.6 \mathrm{GHz}$ frekans bandı WLAN uygulamaları için düşük gürültülü kuvvetlendirici üretimi gerçekleştirilmiştir. LNA devresi BFP720 SiGe Transistörü ve yüzey montaj smd malzemeler kullanılarak üretilmiş ve ölçümler N5234A PNA-L Microwave Network Analyzer kullanılarak elde edilmiştir. Devre $3.3 \mathrm{~V}$ besleme gerilimi ile çalışmaktadır. Sekil 4'de üretilen devrenin baskı devre modeli ve üretilen devre gösterilmiştir. Üretilen LNA devresinin baskı devre gösterimi şekil 4(a) da gösterilmiştir. Devrenin boyut olarak $33 \times 22 \mathrm{~mm}^{2}$ lik alan kaplamaktadır. SMD malzemelerin kılıfları 0805 ve 0603 olarak kullanılmıştır. Şekil 4(b) de üretilen devre verilmiştir. Devre üzerine eleman yerleşimi ve besleme bağlantıları yapılmıştır. Devre üretimi LPKF S63 cihazı kullanılarak bakır kazıma işlemleri yapılmışıtır. Şekil 4(c) de ölçümleri yapılan LNA devresinin port bağlantıları gösterilmiştir.

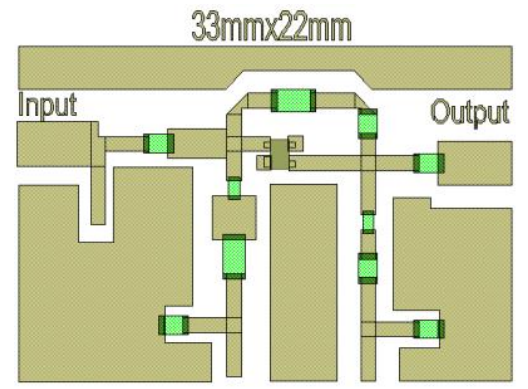

(a)

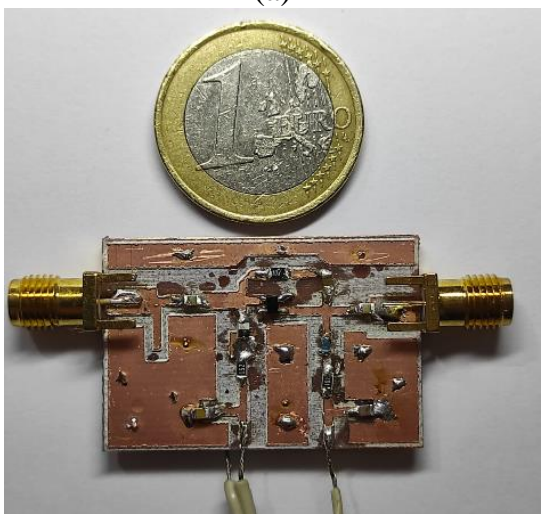

(b)

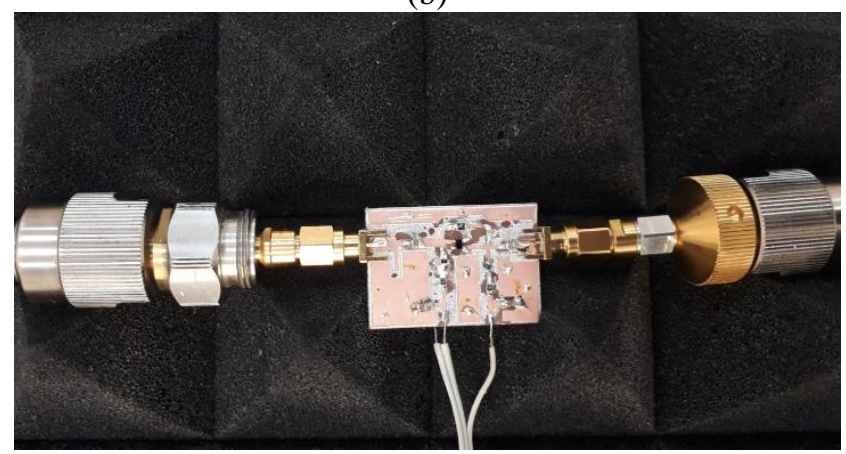

(c)

Şekil.4 WLAN Uygulamaları için Tasarlanan LNA (a) Baskı Devre gösterimi (b) Üretilen Devre (c) Ölçüm port bağlantıları

Tablo 2'den görüldüğü üzere bu çalışmada, WLAN standartları ile uyumlu, yüksek performanslı, düşük maliyetli, alıcı-verici sisteminin alt ünitelerinden LNA tasarımı üzerinde çalışılmıştır. Tablo 2'de LNA devresinin benzetim ve ölçüm sonuçları kıyaslanmıştır. Tasarlanan devrenin kazancı $\sim 13 \mathrm{~dB}$ olarak tasarlanmıştır. Düşük gütültülü kuvvetlendirici devresinde 
yapılan simülasyonlarda devreye $3.3 \mathrm{~V}$ uygulandığında $36.3 \mathrm{~mW}$ güç harcamakta ve $1 \mathrm{~dB}$ sıkışma noktası $-18 \mathrm{dBm}$ olarak elde edilmiştir. LNA boyutu $33 \times 22 \mathrm{~mm}^{2}$ olarak tasarlanmış ve üretilmiştir.

Tablo 2. WLAN Bandı LNA Performans Sonuçları

\begin{tabular}{|lcc|}
\hline & Benzetim & Ölçüm \\
\hline Vcc & 3.3 & 3.3 \\
\hline Çalışma Frekansı [MHz] & 5600 & 5600 \\
& & \\
\hline Gürültü Şekli (dB) & 1.87 & --- \\
\hline Kazanç (dB) & $>14$ & 13 \\
\hline Giriş Geri Dönüş Kaybı (dB) & $<-17$ & $<-15$ \\
& & \\
\hline Çıkış Geri Dönüş Kaybı (dB) & $<-22$ & $<-15$ \\
& & \\
\hline S11 Dip Noktası & $5600 \mathrm{MHz}$ & $5500 \mathrm{MHz}$ \\
\hline Güç Tüketimi & $28.3 \mathrm{~mW}$ & $36.3 \mathrm{~mW}$ \\
\hline Akım Tüketimi & $10 \mathrm{~mA}$ & $11 \mathrm{~mA}$ \\
\hline Kararlılık (K) & $>1$ & $>1$ \\
\hline Boyut (mm) & $33 \times 22$ & $33 \times 22$ \\
\hline
\end{tabular}

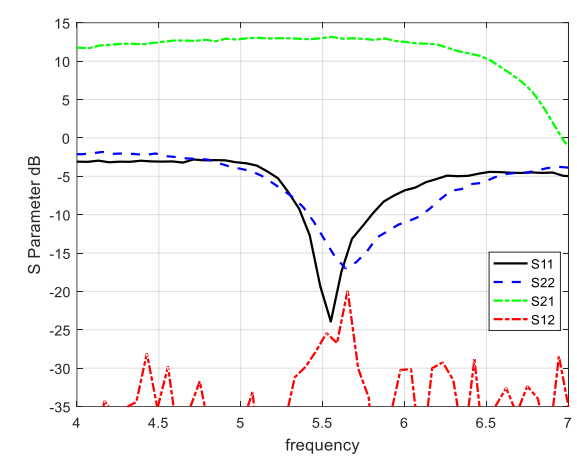

\section{Şekil.5 WLAN Uygulamaları için Üretilen LNA S Parametresi Sonuçları}

Şekil 5'de ürettirilen devrenin $4-7 \mathrm{GHz}$ frekans bandında ölçülen S parametreleri değerleri gösterilmektedir. Giriş ve çıkıştaki geridönüş kaybı $5.6 \mathrm{GHz}$ de $-15 \mathrm{~dB}$ seviyesinin altında ölçülmüştür. İzolasyon değerleri $-20 \mathrm{~dB}$ altında ölçülmüştür. $\mathrm{S}_{21}$ iletim değeri ise $13 \mathrm{~dB}$ olarak ölçülmüştür.

\section{Sonuç}

Bu çalışmada, WLAN standartları ile uyumlu düşük gürültü kuvvetlendirici (LNA) ürettirilmiş ve ölçüm sonuçları verilmiştir. BFP720 SiGe transitörlü LNA tasarımı yapılmıştır. Tasarımda Microwave Office AWR programı yazılımı kullanılarak elde edilmiş olup, ölçümler N5234A PNA-L Microwave Network Analyzer kullanılarak elde edilmiştir. Devrede taban malzemesi olarak FR4 cam elyaf kullanılmıştır. Üretilen devrenin kazanc1 $\sim 13 \mathrm{~dB},-10 \mathrm{~dB}$ band genişliği 5.45$5.7 \mathrm{GHz}$ olarak ölçülmüştür. Düşük gütültülü yükselteç devresinde yapılan simülasyonlar da $1 \mathrm{~dB}$ sıkışma noktası $18 \mathrm{dBm}$ olarak elde edilmiş olup, devre $3.3 \mathrm{~V}$ uygulandığında $36.3 \mathrm{~mW}$ güç harcamaktadır. Tasarlanan DGY'nin boyutu $33 \times 22$ $\mathrm{mm}^{2}$ olarak tasarlanmış ve üretilmiştir.

Sonuç olarak tasarımı ve üretimi gerçekleştirilen düşük gürültülü kuvvetlendiricinin $5.45 \mathrm{GHz}$ ile $5.7 \mathrm{GHz}$ arasında verimli bir şekilde kullanılabileceği görülmektedir. Ayrıca tasarlanan kuvvetlendiricinin az hacim kaplaması ve düşük maliyete sahip olması önemli avantajlarındandır. Üretilen kuvvetlendirici WLAN sistemleri için tasarlanacak modüllerde kullanılarak bilimsel araştırmalarda ve ticari ürün uygulamalarda kullanılmak üzere değişik uygulamalarda kullanılabilecektir.

\section{Kaynakça}

Akyildiz, I F, Su, W, Sankarasubramaniam, Y, Cayirci, E. (2002). Wireless Sensor Networks-A Survey. Elsevier Computer Networks, (38), 393-422.

Alaybeyoğlu, A, Kantarcı, A, Erciyes, K . (2009). Telsiz Duyarga Ağlarında Hedef İzleme Senaryoları. Akademik Bilişim 2009 konferans1, Bildiri No: 69, Harran Üniversitesi, Şanlıurfa.

Çalışkan, A, Kızılay, A, Belen, M, Mahouti, P . (2019). ISM Band Haberleşme Uygulamaları İçin Origami Anten Tasarımı. Avrupa Bilim ve Teknoloji Dergisi , (16) , 785-791 . DOI: 10.31590 /ejosat.573379.

Danac1, H, Palandöken, M . (2020). A Novel Electronically Reconfigurable Antenna Design for RFID and GSM 900 $\mathrm{MHz}$ Applications . Avrupa Bilim ve Teknoloji Dergisi , Ejosat Özel Say1 2020 (ICCEES) , 304-307 . DOI: 10.31590/ejosat.804511.

Demirel, S, Güneş, F, Mahouti, P . (2017). Adjoint sensitivity analysis of the $T, \Pi$, and $L$ types of microstripline low noise amplifiers. Int. J. Numer. Model., (30), e2133. doi: 10.1002/jnm.2133.

Doddamani, N D, Nandi, A V, Chandra, H . (2007). Design of SPDT Switch, 6 Bit Digital Attenuator, 6 Bit Digital Phase Shifter for L-Band T/R Module using 0.7 uM GaAs MMIC Technology. International Conference on Signal Processing, Communications and Networking. ICSCN '07. pp.302307.

Esame, O, Kaynak, M, Kavlak, C, Bozkurt, A, Tekin, I, Gürbüz, Y . (2006). IEEE 802.11a Standard Uyumlu, RF Alıc1-Verici Alt-Blok Devrelerinin Gerçeklenmesi. URSI, Hacettepe Üniversitesi.

Hashemi, H, Hajimiri, A . (2002). Concurrent Multi-Band LowNoise Amplifiers Theory, Design and Applications, IEEE Trans. Microwave Theory and Techniques, (50), no. 1, pp. 288-301.

Hove, C, Faaborg, J . (2004). $0.35 \mu \mathrm{m}$ CMOS T/R Switch for 2.4 GHz Short Range Wireless Applications., Analog Integrated Circuits and Signal Processing, (38),pp. 35-42.

https://www.infineon.com/cms/en/product/rf-wireless-control/rftransistor/ultra-low-noise-sigec-transistors-for-use-up-to-12ghz/bfp720/

Kluge, W, Dathe, L, Jaehne, R, Ehrenreich, S, Eggert, D . (2003). A 2.4GHz CMOS Transceiver for $802.11 \mathrm{~b}$ Wireless LANs. IEEE ISSCC Dig. Tech. Papers, pp. 360-361.

Koçer, M , Aydemir, M . (2020). Microstrip Patch Antenna Design for Military Satellite Communication . Avrupa Bilim ve Teknoloji Dergisi , Ejosat Özel Sayı 2020 (ICCEES), 142-147 . DOI: 10.31590/ejosat.801959.

Stece, C. (1999). RF Power Amplifiers for Wireless Communications, Artech House.

Mahouti, P , Güneş F, Demirel, S . (2012). Honey bees mating algorithm applied to feasible design target space for a wideband front- end amplifier. 2012 IEEE International Conference on Ultra-Wideband, pp. 251-255, doi: 10.1109/ICUWB.2012.6340411.

Pozar, D. M. (1998). John Wiley\&Wiley, Microwave Engineering.

Ulrich, L R . (2000). John Wiley\&Sons, RF/Microwave Circuit Design For Wireless Applications. 